\title{
Enseignement de la langue et apprentissage de la citoyennté
}

\section{Bernard Combettes}

\section{(2) OpenEdition}

1 Journals

Édition électronique

URL : http://journals.openedition.org/trema/1701

DOI : 10.4000/trema.1701

ISSN : 2107-0997

\section{Éditeur}

Faculté d'Éducation de l'université de Montpellier

\section{Édition imprimée}

Date de publication : 1 octobre 1999

Pagination : 41-50

ISSN : 1167-315X

\section{Référence électronique}

Bernard Combettes, «Enseignement de la langue et apprentissage de la citoyennté », Tréma [En ligne], 15-16 | 1999, mis en ligne le 26 février 2013, consulté le 19 avril 2019. URL : http:// journals.openedition.org/trema/1701 ; DOI : 10.4000/trema.1701

Ce document a été généré automatiquement le 19 avril 2019

Trema 


\title{
Enseignement de la langue et apprentissage de la citoyennté
}

\author{
Bernard Combettes
}

1 Mettant à profit la parution récente de nouveaux programmes pour l'enseignement du français au collège, nous examinerons essentiellement, dans les lignes qui suivent, les possibilités offertes par ces essais de rénovation lorsqu'il s'agit de mettre en relation le travail sur la langue et l'apprentissage de la citoyenneté.

\section{Quelques éléments de mise en perspective historique}

2 Il ne semble cependant pas inutile, avant de développer quelques points particuliers rattachés à cette thématique de rappeler que la question du langage, de son importance et de son rôle, est toujours présente - plus ou moins explicitement - chez les penseurs qui se sont attachés à définir la citoyenneté. Une approche historique permet de constater rapidement que les conceptions de ce rapport, de cette relation, entre le domaine du langage et celui de la citoyenneté, sont loin d'être identiques; les différences n'ont ici rien de surprenant, étant donné d'une part, que la notion même de citoyenneté est loin de faire l'unanimité, et d'autre part, que la nature et les fonctions du langage peuvent être approchées sous des angles fort divers : une conception du langage comme moyen de « représentation » n'autorise évidemment pas les mêmes conclusions qu'une conception qui privilégie la dimension «expressive » ou la dimension «communicative». On peut cependant noter que, dès l'Antiquité, apparaissent les principales idées qui seront reprises et développées par la suite. Ainsi le début de la Politique d'Aristote souligne-t-il parfaitement la nécessaire liaison, quasiment imposée de façon logique, pourrait-on dire, qui s'institue entre le langage et la citoyenneté; il nous semble intéressant de citer intégralement le passage où se développe une argumentation fortement structurée, qui conduit des caractéristiques «naturelles» à la vie en société, et qui fait du langage l'instrument spécifique de cette relation: 
"[...] La nature ne fait rien en vain; or seul parmi les animaux l'homme a un langage. Certes, la voix est le signe du douloureux et de l'agréable, aussi la rencontre-t-on chez les animaux; leur nature, en effet, est parvenue jusqu'au point d'éprouver la sensation du douloureux et de l'agréable et de se les signifier mutuellement. Mais le langage existe en vue de manifester l'avantageux et le nuisible, et par suite aussi le juste et l'injuste. Il n'y a en effet qu'une chose qui soit propre aux hommes par rapport aux autres animaux: le fait que seuls ils aient la perception du bien, du mal, du juste, de l'injuste et des autres notions de ce genre. Or avoir de telles notions en commun, c'est bien ce qui fait une famille et une cité." (Aristote : Politique,Livre I, Ch. 2).

3 La citoyenneté se caractérise avant tout par la mise en commun de certaines valeurs, et l'expression de ces valeurs passe obligatoirement par le langage; à la différence des simples « sensations ", qui peuvent se traduire par des cris, les notions telles que celles du juste et de l'injuste doivent dépasser le stade de l'intuition première pour emprunter la voie d'une expression structurée et élaborée. La cité ne se construit pas à partir de la mise en commun d'intérêts qui demeureraient au niveau de la sensation - en ce sens, il n'y aurait guère de différence entre les collectivités d'animaux et celles des hommes - mais à partir d'une communauté de valeurs qui se trouvent indissociablement liées à l'activité langagière.

Une dimension supplémentaire, et importante pour le thème qui nous intéresse ici, est introduite par Platon qui, tout en reprenant la notion de mise en commun des valeurs, ajoute celle de "pudeur » $\alpha_{1} \delta \circ$ (aidos) : ce qui est partagé de façon égale entre les hommes, ce n'est pas seulement la justice, mais aussi une certaine façon de tenir compte $\mathrm{du}$ jugement d'autrui. Le citoyen doit respecter les lois mais ce respect, ou, plus exactement, la justification de ce respect, s'opère par une mise en rapport avec le regard des autres. Il ne s'agit donc plus uniquement de la nature des relations qui s'instaurent entre les citoyens, mais aussi et surtout de la manière dont ces relations sont établies, mise en œuvre. On rappellera à ce propos que le terme de « civilité », avant de s'affaiblir pour désigner une certaine forme de politesse, conservait sa valeur étymologique et renvoyait à l'aptitude à vivre en commun, à être sociable, le latin civilis étant un calque du

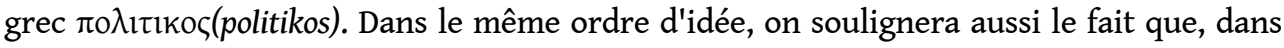
la mythologie grecque, la « persuasion » $\pi \varepsilon 1 \theta o \varsigma$ (peithos) est d'ordinaire accompagnée de

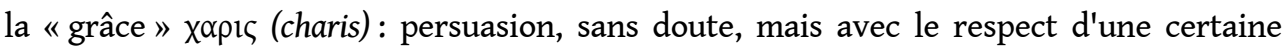
forme, qui autorise la mise en commun.

De telles conceptions débouchent de façon naturelle sur la notion de «lieu de discussion ", notion abondamment reprise par les modernes; il suffira de renvoyer ici à l'importance accordée par H. Arendt au concept d'espace public, à la description qu'elle donne de l'agora grecque comme lieu essentiel au projet politique, ou encore à l'opposition entre espace privé et espace public telle qu'elle est élaborée et exploitée par J. Habermas.

6 Le langage, dans son rapport à la citoyenneté, apparaît donc comme d'abord instrument de mise en œuvre de l'esprit critique, dans la mesure où il s'agit d'exprimer des valeurs qui seront partagées, mais il est également une forme de pouvoir, puisque la mise en commun des valeurs s'accompagne de l'activité de persuasion, de la participation aux délibérations, aux décisions, autant de réalisations qui vont de pair avec une certaine « forme » du discours, ainsi qu'avec l'existence d'un lieu de discussion, de dialogue. 


\section{Maîtrise du langage et participation à la vie publique}

7 La conception ainsi rapidement résumée ne doit cependant pas faire oublier une autre approche, qui ne met pas l'accent sur le rôle du langage dans l'expression des valeurs ou dans l'acte de persuasion, mais qui insiste sur la maîtrise du langage comme préalable indispensable à la participation à la vie publique; indépendamment de la question de l'expression des valeurs, les compétences linguistiques peuvent être un facteur d'inégalité et interdire la «mise en commun ». Un auteur comme Condorcet illustre bien ce type de raisonnement, qui est sans doute plus «moderne » que celui que nous avons décrit plus haut; dans son Rapport à l'Assemblée Législative (1793), il souligne fréquemment que l'absence d'égalité linguistique empêche la discussion, le débat. Ainsi, l'enseignement de la langue devrait-il, par une amélioration des compétences, conduire à une certaine égalité :

"[...] Les enfants acquerront avec aussi peu de peine les connaissances grammaticales ou d'orthographe nécessaires pour que la langue et l'écriture de la généralité des citoyens se perfectionnent peu à peu; et il est important pour le maintien de l'égalité réelle, que le langage cesse de séparer les hommes en deux classes. »(Condorcet).

Ceux qui savent le français peuvent entrer en communication avec tout le monde, alors que les autres se trouvent exclus de la vie politique, étrangers à la cité. Dans de nombreux cas, d'ailleurs, plutôt que d'exclusion, il s'agit de dépendance, ce qui a un résultat identique en ce qui concerne la non-participation à la citoyenneté :

"Celui qui a besoin de recourir à un autre pour écrire ou même lire une lettre, pour faire le calcul de sa dépense ou de son impôt, pour connaître l'étendue de son champ ou le partager, pour savoir ce que la loi lui permet ou lui défend; celui qui ne parle point sa langue de manière à pouvoir exprimer ses idées, qui n'écrit pas de manière à être lu sans dégoût ; celuilà est nécessairement dans une dépendance individuelle, dans une dépendance qui rend nul ou dangereux pour lui l'exercice des droits du citoyen. " (Condorcet - Cité par, BRUNOT F. : Histoire de la Langue Française. Tome IX, pp. 101-102).

9 Cette exigence de ce que l'on pourrait appeler une "autonomie» linguistique n'est évidemment pas en contradiction avec les définitions proposées par Aristote ou par Platon; la mise en commun des valeurs demeure au niveau de l'utopie si les conditions qui peuvent permettre les échanges ne sont pas remplies.

\section{Comment les instructions officielles posent-elles le problème?}

10 Les textes officiels actuellement en vigueur dans l'enseignement secondaire prennent en compte les divers aspects de cette problématique, d'une façon peu homogène ; en effet, alors que les nouvelles instructions pour le collège insistent sur la capacité à s'exprimer : " approchant de l'âge de la majorité, les élèves deviennent des participants actifs de la vie sociale: ils doivent donc être tous en mesure de s'exprimer et de structurer leur jugement " (Programmes, p. 17), les documents d'accompagnement mettent l'accent sur la maitrise des discours, maîtrise qui concerne à la fois, semble-t-il, les activités de production et celles de compréhension :

"[...] La formation du citoyen s'impose comme une finalité majeure. Pour l'enseignement du français, cette finalité se traduit par un objectif central, la maîtrise des discours [...] Si les objectifs des programmes précédents - savoir s'exprimer, acquérir une culture, accéder à la 
méthode et à l'autonomie - gardent bien entendu toute leur pertinence, ils sont désormais régis par l'apprentissage des formes de discours. Cet apprentissage donne aux acquisitions leur orientation générale et les situe dans leur contexte." (Accompagnement des

Programmes de $6^{e}$, p. 11).

11 Il ne parait pas impossible, bien que les enchaînements qui conduisent d'un argument à l'autre demeurent quelque peu implicites, de voir dans ces recommandations la trace des deux grandes directions de pensée que nous venons d'évoquer, la nécessité de disposer d'un instrument, le langage, qui permette à la fois l'expression et la structuration du jugement, se trouve en quelque sorte subordonnée aux «conditions» dans lesquelles cette expression se réalise. La priorité donnée aux "formes de discours" peut être interprétée comme le souci, sans aucun doute louable, de ne pas s'en tenir à l'apprentissage du "savoir s'exprimer », mais de prendre en compte les situations de production, ce qui renvoie à une conception du langage comme pouvoir, en particulier dans les activités de persuasion, d'argumentation.

\section{L'introduction de la notion de discours et ses conséquences}

12 En commençant par cette notion centrale de "discours ", nous tenterons à présent de cerner, dans les textes officiels, mais aussi à travers les pratiques courantes qui reflètent bien souvent des approches encore traditionnelles, les principaux points, les principaux domaines, à partir desquels devrait s'organiser une réflexion sur les rapports étude de la langue/citoyenneté, réflexion qui pourrait avoir des conséquences sur les contenus et plus encore sur les méthodes de l'enseignement de la langue.

13 L'apprentissage du discours peut être considéré comme une avancée importante, dans la problématique qui nous intéresse ici, par rapport aux contenus antérieurs, qui avaient laissé une place aux aspects textuels, mais faisaient courir le risque d'une étude de la langue et des textes un peu trop refermée, limitée, consacrée uniquement à l'analyse des caractéristiques formelles, sans qu'il y ait une prise en compte réelle des contextes de situation. Dans les instruction actuelles, l'ensemble de l'enseignement de la langue apparaît donc comme « subordonné », dans le bon sens du terme, à l'approche discursive : qu'il s'agisse de la morphosyntaxe ou des marques qui assurent la cohérence textuelle, les faits de langue devraient être systématiquement abordés dans leur relation avec les formes de discours, et, par là même, devraient toujours être replacés dans un cadre qui permette de rendre compte de leur rôle dans l'interaction, de la dimension sociale des phénomènes linguistiques.

14 Ce principe général ne peut guère être critiqué, même s'il laisse entier un problème à nos yeux fondamental : cette priorité accordée au discours apportera-t-elle des améliorations à l'apprentissage du système de la langue? L'enrichissement des compétences en "grammaire de phrase » - enrichissement indispensable, rappelons-le, pour qu'une véritable citoyenneté puisse s'exercer - s'opère-t-il obligatoirement par une prise en compte du niveau discursif? Hypothèse implicitement adoptée par les nouveaux programmes, l'interaction, dans les apprentissages, des divers niveaux d'observation (phrase, texte, discours) reste à valider. L'étude du discours elle-même n'est malheureusement pas conçue dans une optique qui serait en cohérence avec les objectifs généraux ; en effet, la dimension sociale, inhérente à l'analyse discursive, se trouve peu prise en compte, du moins dans les premières classes du collège, s'appuyant sur une distinction qui aurait mérité discussion, entre les «effets» (convaincre, émouvoir, 
persuader, etc) et les «fonctions» (raconter, décrire, expliquer, justifier, argumenter, etc), le texte des instruction "écarte le critère des effets, plus difficile à analyser, parce qu'il fait appel à des éléments du contexte social (par exemple, ce qui peut convaincre les uns ne convaincra pas les autres)" (Compléments, p. 12). Dans une telle perspective, on ne perçoit plus l'intérêt d'une séparation nette entre le texte et le discours si l'étude de ce dernier se réduit à une observation des caractéristiques "internes " qui déterminent un "type " d'énoncé, sans qu'il y ait une véritable réflexion sur la mise en situation réelle et concrète de l'activité langagière. L'occultation de cette dimension conduit ainsi à s'intéresser à quelques aspects limités du contexte situationnel : étude des déictiques, par exemple, ou de l'opposition récit/discours ; mais le domaine sociolinguistique n'est pour ainsi dire pas explicité : la mention des traditionnels «niveaux de langue » ne peut évidemment pas constituer un point d'ancrage pertinent et satisfaisant.

Apprendre à communiquer autour du livre.

Le Courrier de I'UNESCO. Paris, UNESCO, Juillet 1990 (Vol. 43, № 7), 50 p., p. 39.

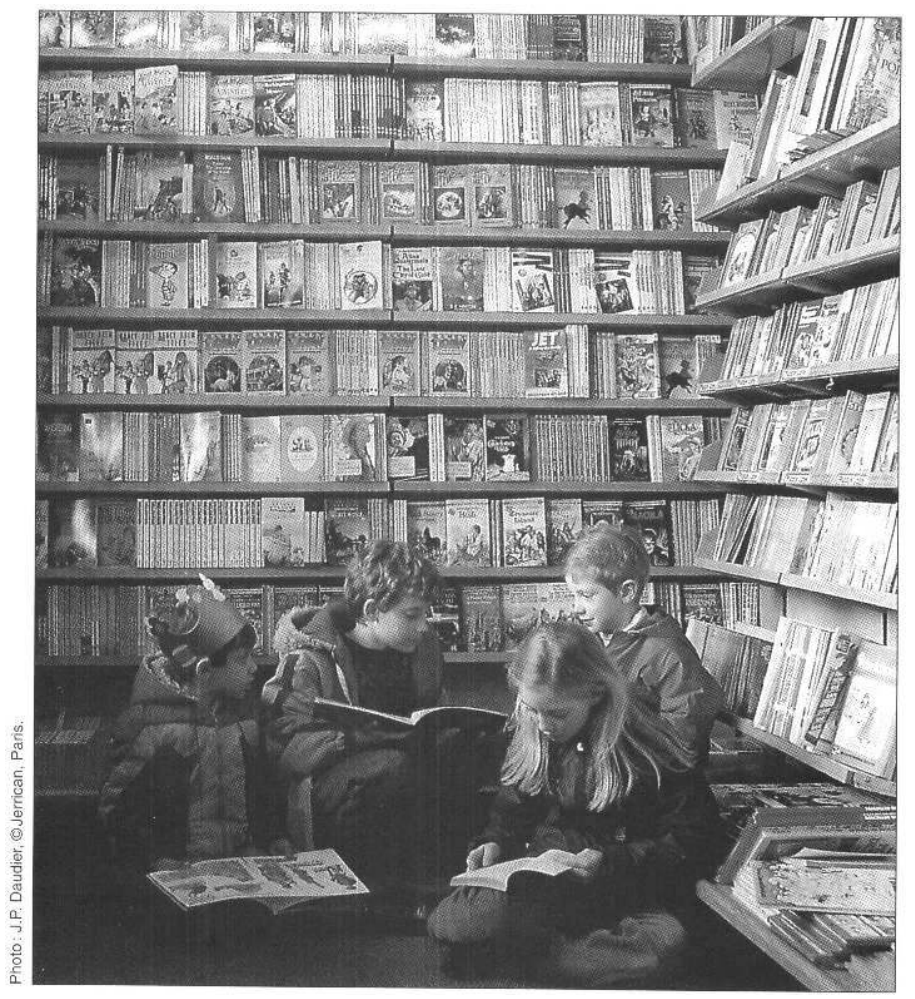

Le travail sur le discours ne doit pas se concevoir sans une réflexion sur les lieux où s'exerce ce discours, sans une observation du fonctionnement des diverses forces qui régissent les places des acteurs de l'interaction. Tout ceci soulève de nombreuses difficultés, mais on ne peut pas prendre comme argument, ainsi que le fait le texte des instructions, la « difficulté » d'un type d'approche pour éliminer ce qui constitue en fait la spécificité du cadre proposé ! Comment travailler sur l'argumentation en ne prenant en compte que sa forme et non sa visée ? Ne serait-il pas possible, pour éviter l'artifice de certaines "mises en contexte ", de prendre appui sur la situation scolaire elle-même, qui fournit assez de matière à la mise en place de notions et de concepts permettant l'approche du fonctionnement social des échanges? Quelle que soit la démarche didactique privilégiée, il parait essentiel d'élargir le champ d'observation en ouvrant l'éventail de toutes les possibilités offertes par les diverses réalisations écrites: qu'il 
s'agisse des sujets d'examen portant sur l'argumentation ou d'exercices comme celui de la "lecture méthodique", propositions qui ont pu apparaître comme des essais de rénovation d'une démarche traditionnelle, le glissement vers une application systématique au texte littéraire a été immédiatement perceptible. Étudier les formes de discours implique une prise en compte de toutes les variétés de textes. Se pose alors la question de la place de l'oral et des outils indispensables pour analyser ce type de communication. Les textes officiels ont certes toujours insisté sur l'importance du discours oral, mais on constate que les rares propositions concrètes en ce domaine ne rendent pas bien compte de la spécificité des faits étudiés, qu'il s'agisse du système de la langue parlée ou des situations mêmes de production. Trop souvent considéré comme une "déformation» de l'écrit, examiné avec des concepts qui, s'ils sont à peu près satisfaisants pour la description de la langue écrite, ne peuvent rendre compte des caractéristiques particulières de la langue parlée, l'oral demeure le parent pauvre de l'enseignement du français; si l'on veut donner au discours la place qui lui revient, il n'est pas possible de se contenter d'une telle limitation du champ d'observation.

\section{Reconsidérer le rapport à la norme}

16 D'une façon plus générale, c'est toute une attitude face au problème de la norme et de la variation qu'il convient d'adopter: l'approche discursive ne peut aller de pair avec une idéologie du «bon usage » qui conduit à considérer que les « niveaux de langue » sont une réalité justifiée par le système même de la langue, que des exigences telles que celles de la « clarté » ou de la « beauté » de l'expression entraînent l'emploi d'un registre " correct », voire soutenu. Ce type d'affirmation masque le fait que la norme est de nature sociale et non de nature linguistique, et que ce n'est pas la forme d'une expression qui justifie son statut, sa position sur l'échelle des valeurs "sociolinguistiques». La relation à établir n'est d'ailleurs pas une relation directe qui réunirait une variante et une convenance sociale ; il est nécessaire de prendre en compte un certain degré d'adaptation des formes aux activités d'encodage, aux liens avec le domaine cognitif. Plutôt que l'objectif « maîtriser la langue », qui demeure très éloigné des réalités discursives, c'est l'objectif «maîtriser les structures variationnelles du système linguistique» qui devrait être assigné à un enseignement soucieux d'une prise en compte réelle du discours. Se pose alors, dans un enchaînement logique, la question des outils qui permettent d'aborder correctement la description de la variation; nous n'entrerons pas ici dans les aspects techniques de ces problèmes, nous contentant de rappeler que certaines théories renvoient la variation à l'exceptionnel, au marginal, alors qu'il serait nécessaire de la traiter au contraire comme centrale, inhérente au système même de la langue. C'est la structure de ce système qui permet le "jeu», les degrés, les progressions, dans l'application des diverses règles ; travailler sur les grammaires « floues » aurait l'avantage d'échapper à une certaine rigidité due aux approches distributionnelles qui ont inspiré en grande partie la rénovation de l'enseignement grammatical depuis une trentaine d'année.

Donner toute sa place à la variation, c'est aussi prendre en compte la diachronie ; certes, il ne s'agit pas obligatoirement d'envisager une diachronie longue, qui décrirait les diverses étapes du français, mais plutôt de donner les bases d'une réflexion sur l'évolution des faits de langue, de faire prendre conscience que le système linguistique est constamment soumis au changement : la dimension temporelle ne peut être négligée, elle permet de comprendre comment telle expression peut cohabiter avec telle autre, 
comment elle va se trouver minorée ou, au contraire valorisée, etc. Il serait souhaitable que l'approche linguistique rejoigne ici les principes souvent énoncés lorsqu'il s'agit de l'aspect culturel de l'enseignement du français, principes qui soulignent l'importance des données du passé pour une bonne compréhension de la culture d'aujourd'hui.

Un examen des nouveaux programmes fait donc apparaître, dans la partie des contenus ainsi que dans les illustrations "pratiques", l'absence d'une exploitation véritable de l'approche discursive, approche pourtant indispensable si l'on désire établir un lien entre l'étude de la langue et le domaine de la citoyenneté. Il faut aussi remarquer que la sémantique, éternel parent pauvre des programmes scolaires, est encore une fois sacrifiée : les avancées de la linguistique n'ont guère eu de répercussions dans cette partie de l'enseignement $d u$ français. Pourtant, les études d'analyse du discours sont essentiellement lexicales et c'est d'ailleurs par là qu'il faudrait commencer, nous semble$\mathrm{t}$-il, dans des tentatives de transposition didactique. Ici encore, un objectif tout à fait défendable ("ouvrir, le plus souvent possible, une perspective de saisie sociale») ne se trouve pas réellement exploité par des contenus qui demeurent traditionnels et n'offrent guère, dans les notions proposées ("synonymes, doublets, antonymes, composition, étymologie »), la possibilité de travailler sur la dimension discursive. Ces insuffisances en ce qui concerne la sémantique lexicale se doublent d'une absence quasiment totale d'outils permettant d'aborder ce que l'on pourrait appeler la sémantique référentielle. Il est difficile de séparer l'étude du discours, des problèmes liés à la « connaissance partagée », aux divers types de désignations, aux différents modes de donation du référent. Rien dans les textes officiels ne suscite une réflexion sur ces points fondamentaux, réflexion sans laquelle il semble vain de proposer une analyse des faits de langue qui prenne vraiment en compte l'interaction sociale où s'exerce l'activité discursive.

\section{Poursuivre le mouvement de réforme des programmes et des concours}

Ce tour d'horizon rapide aboutit à une constatation relativement simple : la rénovation actuelle des programmes établit des objectifs généraux qui affirment clairement l'importance de l'étude de la langue pour ce que l'on pourrait appeler l'apprentissage de la citoyenneté, et la priorité accordée au discours, dans une telle perspective, est parfaitement logique ; cet aspect des textes marque un très net progrès par rapport aux précédentes instructions. Malheureusement, un certain manque de cohérence avec les contenus proposés, le maintien de notions et de concepts qui reflètent davantage la tradition qu'un vrai changement, la mise à l'écart des principaux acquis de la linguistique durant ces vingt dernières années, font que ces objectifs ne peuvent se traduire au niveau didactique et qu'il y a de fortes chances pour que le changement, s'il se produit, n'affecte guère l'apprentissage de la citoyenneté.

Ce constat pessimiste n'est pas modifié, bien au contraire, par un examen de la situation en ce qui concerne la formation initiale des enseignants. Si on considère en effet les concours de recrutement, qui influencent, dans une grande mesure, les études universitaires, la formation actuelle apparaît comme inadaptée, dans la mesure où elle n'intègre quasiment pas les points que nous venons d'évoquer. On pourrait certes considérer que les concours évaluent un savoir "académique", et que l'année de formation est destinée à la réflexion sur les aspects didactiques. Mais la formation sur le 
discours, sur la sociolinguistique, ne peut être assimilée à une simple question d'application, une simple sous-partie de la linguistique appliquée, comme s'il s'agissait d'observer le fonctionnement en situation d'un système linguistique que l'on aurait d'abord examiné sous un angle « théorique ». La formation en ces domaines est du même ordre, et de la même importance que celle qui est donnée pour la littérature et pour les autres disciplines. Les concours de recrutement pour les professorats de lettres sont le reflet d'une conception de la formation qui date des années soixante; si l'on excepte l'introduction récente de l'épreuve de didactique à l'oral, le CAPES de lettres modernes demeure construit sur le modèle de l'ancienne licence d'enseignement, qui était composée de quatre certificats et se déroulait sur deux années. Tant en ce qui concerne la structure des concours (nombre, nature, importance des diverses disciplines et sousdisciplines représentées) qu'en ce qui relève des contenus et des méthodes évalués, il est urgent de procéder à une rénovation profonde : il serait tout à fait illogique, et inefficace, de toucher aux programmes scolaires sans s'occuper en même temps de la formation des maîtres.

\section{RÉSUMÉS}

Non disponible

Not available

\section{AUTEUR}

BERNARD COMBETTES

Université de Nancy II 